Ann. Zootech., I973, 22 (3), 295-301.

\title{
OBSERVATIONS SUR L'ANCESTRUS POST-PARTUM DES BREBIS ROMANOV APRÈS UN AGNELAGE EN SAISON SEXUELLE
}

\author{
I. TCHAMITCHIAN, G. RICORDEAU, C. LEFÈVRE et A. DESVIGNES \\ avec la collaboration technique de $\mathrm{D}$. Lajous \\ Laboratoire de Génétique des Petits Ruminants, \\ Centre de Recherches de Toulouse, I. N.R. A., \\ B. P. 12, 31320 Castanet Tolosan
}

\section{RÉSUMÉ}

Sur 46 brebis Romanov en rythme accéléré de reproduction (Tсhamitchian et al, 1973), ayant mis bas du 18-09 au 23-10-1969, et séparées de leurs agneaux quelques jours après, nous avons contrôlé les venues en cestrus et effectué les saillies sur toutes les chaleurs observées jusqu'au 20-II-I969; 2 brebis n'ont jamais manifesté d'œstrus.

Les premiers œestrus apparaissent i I à 47 jours après la mise bas ( 24 jours en moyenne sur 44 brebis), mais ils sont peu fertiles (fig. I). D'autre part, la distribution des intervalles entre les deux premiers œstrus et entre le deuxième et le troisième, fait apparaitre des cycles courts de muins de I I jours et des cycles normaux de I 3 à 20 jours (fig. I). Les saillies après un cycle court sont moins fécondes qu'après un cycle normal (tabl. I); aussi les fécondations se situent-elles 20 à 50 jours après la mise bas ( 36 jours en moyenne), mais essentiellement entre 34 et 45 jours.

L'intervalle mise bas-I er ostrus ou mise bas-fécondation varie de façon significative avec la date de mise bas pour la période considérée, ce qui semble indiquer que l'anœstrus post-partum est minimum après les mises bas d'octobre (tabl. 2).

L'accélération du rythme de reproduction des brebis dépend de leur fertilité au cours de 1'année, mais aussi de la reprise du comportement cyclique et de l'ovulation après la mise bas.

Dans le cadre d'une expérimentation concernant des brebis soumises à 4 périodes de lutte en 2 ans (Tchamitchian et al., I973), nous avons étudié l'apparition de l'œstrus et la fertilité des brebis après la mise bas en saison sexuelle. Cette étude présente les résultats obtenus sur 46 brebis adultes Romanov ayant agnelé en septembre-octobre Ig69. 


\section{CONDITIONS EXPÉRIMENTAL,ES}

Les 46 brebis considérées ont agnelé du 18-09 au 23-I0-I969 après une durée de gestation normale. Sur cet effectif, 44 avaient déjà mis bas du 25-I2-68 au 15-02-I969. Ces brebis ont été séparées de leurs agneaux $4^{8}$ heures après l'agnelage et ont été ensuite soumises à la détection de l'oestrus après un délai de 5 à ro jours nécessaire à leur tarissement. Les saillies ont été effectuées sur tous les ostrus observés, du 20-09 au 23-II-I969, c'est-à-dire que la période de lutte n'a pas été de même durée pour toutes les brebis : 2 mois pour les premières brebis ayant mis bas et seulement I mois pour les dernières.

\section{Définitions}

Taux de fertilité : brebis mettant bas en $\mathrm{p}$. Ioo des brebis mises en lutte.

Taux de fécondation ou de mise bas: brebis mettant bas en p. roo des brebis saillies.

\section{RÉSULTATS}

\section{I. - Apparition des ostrus}

Sur les 46 brebis ayant mis bas, 44 seulement sont venues en chaleurs II à 47 jours post-partum, soit 24 jours en moyenne. La distribution de la durée du cycle entre le premier et le deuxième oestrus pour les brebis non gravides au premier os-

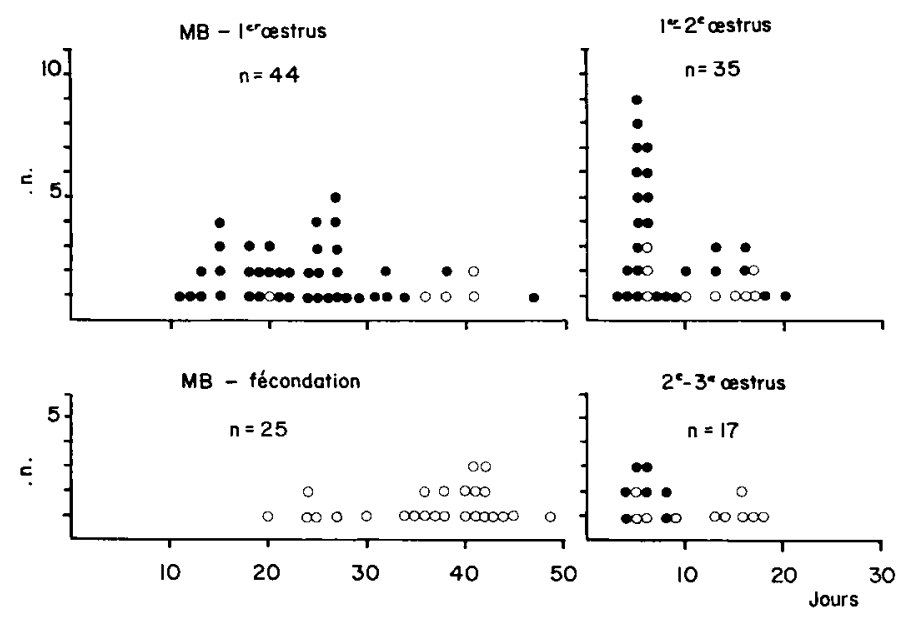

FIG. I. - Activité sexuelle après la mise bas en automne I969

- Estrus

- Fécondation

trus (35 observations) fait apparaître 2 populations de cycles : des cycles courts de Io jours et moins et des cycles apparemment normaux de I3 à 20 jours. Ces deux populations sont également observées entre le deuxième et troisième œstrus sur I7 brebis non encore fécondées au deuxième œstrus (fig. I). 


\section{2. - Fécondations}

Au premier cestrus, sur 44 brebis saillies, 5 seulement sont gravides, soit II,4 p. IOo ; ces brebis ont d'ailleurs un intervalle mise bas- ${ }^{\text {er }}$ cestrus plus long que celui des autres brebis non gravides (fig. I). Au deuxième œstrus, le taux de mise bas est de 16,7 p. Ioo après un cycle court et de 45,4 p. Ioo après un cycle normal ; au troisième cestrus, il est respectivement de 36,4 et Ioo,o p. Ioo (tabl. I). La plupart des saillies fécondantes ont donc bien lieu après un cycle normal, de sorte que les 25 fécondations réalisées se situent finalement 20 à 50 jours après la mise bas $(36$ jours en moyenne), mais essentiellement entre 34 et 45 jours.

\section{TABLEAU I}

Pourcentage de mises bas correspondant aux saillies effectuées sur les premiers cestrus

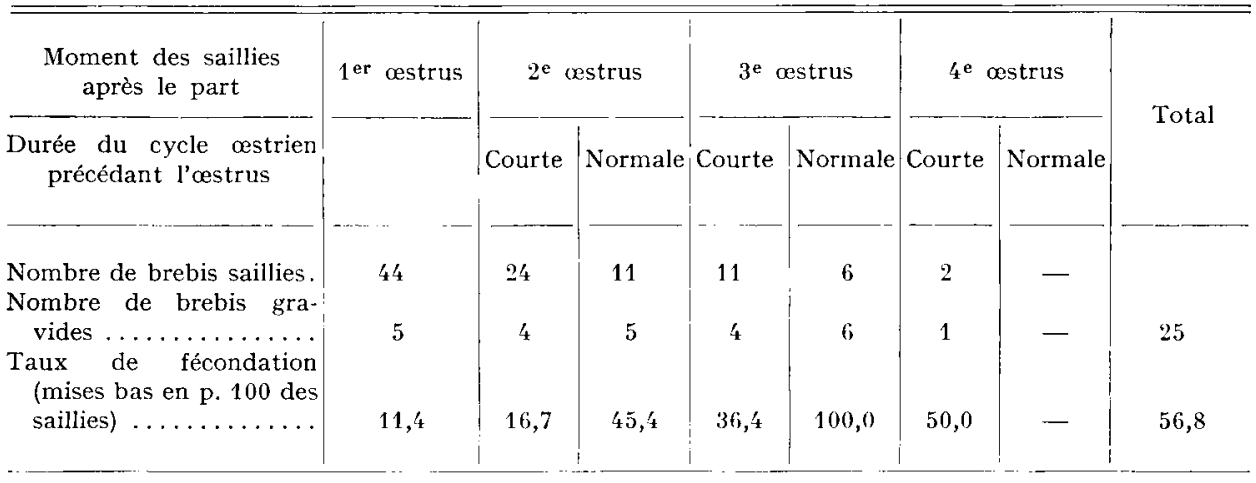

Durée du cycle précédent les $2 \mathbf{e}, 3^{\mathbf{e}}$ et $4^{\mathbf{e}}$ ostrus $\left\{\begin{array}{l}\text { cycle court } \leqslant 10 \text { jours } \\ \text { cycle normal } 13 \text { à } 20 \text { jours. }\end{array}\right.$

Deux brebis n'étant jamais venues en chaleurs, on a seulement 25 brebis gravides sur 46 brebis mises en lutte $(54,3$ p. 10o).

Le faible taux de fertilité enregistré (54,3 p. roo, tabl. I) s'explique également par le fait que la lutte a été arrêtée trop tôt, notamment pour les brebis ayant mis bas le plus tardivement; cependant, la plupart des brebis saillies mais non gravides ont eu au moins 2 cestrus.

\section{3. - Infuence de la date de mise bas précédente}

L'intervalle mise bas-I er œstrus varie de façon significative avec la date de mise bas $\left(b_{1}=-0,54\right)$, ainsi que l'intervalle mise bas-saillie fécondante $\left(b_{2}=-0,45\right)$, mais les deux régressions $\left(b_{1}\right.$ et $\left.b_{2}\right)$ ne sont pas significativement différentes (tabl. 2 , fig. 2). D'après notre échantillon, 1'anœstrus post-partum est done minimum après les mises bas d'octobre, cependant, la période de mise bas (35 jours) et la période effective des saillies sont trop limitées pour qu'il soit possible d'estimer de façon précise la date à laquelle 1'intervalle mise bas-fécondation est minimum. 


\section{TABLEAU 2}

Relation entre la date de mise bas $(x)$ et l'intervalle post-partum $(y)$

\begin{tabular}{c|c|c|c|c|c}
\hline \hline Intervalle post-partum & $n$ & $r$ & $b y, x$ & $s_{b}$ & Équation de régression \\
\hline Mise bas-premier cestrus $\left(y_{1}\right)$ & 44 & $-0,58$ & $b_{1}=-0,54$ & 0,11 & $y_{1}=-0,54 x+41,6$ \\
Mise bas-fécondation $\left(y_{2}\right)$ & 25 & $-0,53$ & $b_{2}=-0,45$ & 0,15 & $y_{2}=-0,45 x+50,2$ \\
\hline
\end{tabular}

La date de mise bas prise comme origine est celle du $1^{\mathrm{er}}$ septembre.

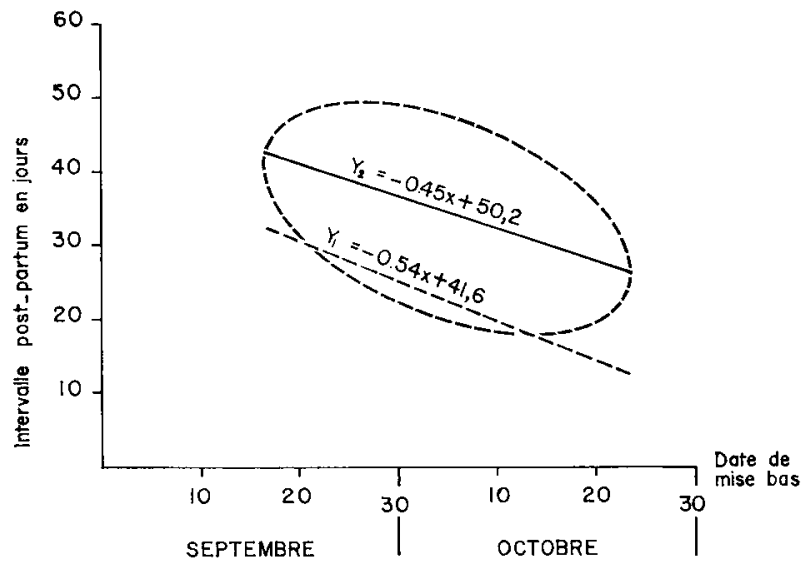

FIG. 2. - Relation entre la date de mise bas, l'intervalle mise bas-fécondation et l'intervalle mise bas-premier cestrus

Intervalle post partum

$y_{1}=$ intervalle mise bas - premier ostrus

$y_{2}=$ intervalle mise bas - fécondation

\section{DISCUSSION}

I $^{\circ}$ Avec les brebis Romanov, en saison sexuelle et après séparation des agneaux, le premier ostrus apparaît en moyenne 24 jours après la mise bas, mais il est peu fertile et les premiers cycles sexuels sont irréguliers, de sorte que les saillies fécondantes se situent en moyenne au $36^{\mathrm{e}}$ jour post-partum. Ce résultat confirme les observations des auteurs russes selon lesquelles les brebis de cette race viennent en chaleurs 30 à 40 jours après la mise bas et peuvent être fécondées aussitôt (DESVIGNEs, I97I). Il est intéressant de remarquer que l'intervalle minimum entre la mise bas et le premier œestrus semble être inférieur à 20 jours pour une mise bas d'octobre, alors qu'avec les brebis Ile-de-France par exemple, l'intervalle mise bas-I er ostrus est indépendant de la date de mise bas lorsque celle-ci a lieu de septembre à novembre et se situe en moyenne à 53 jours (MAUlíon et DAUzrER, I965) : dans ce dernier cas, il s'agit cependant de brebis allaitantes. 
$2^{\circ}$ L'activité sexuelle post-partum dépend de la race et de l'individu, mais aussi de nombreux facteurs non génétiques de variation (mois de mise bas, présence ou absence des agneaux, fréquence des tétées, effet bélier,... cf. Hunter, I968,) Pour expliquer les variations dans "l'expressivité " de cette activité et sans vouloir refaire la bibliographie complète de ce sujet, il est intéressant de résumer ici les principales observations susceptibles d'éclairer nos propres résultats. Il importe tout d'abord de distinguer les chaleurs post-partum, qui arrivent moins de 48 heures après la mise bas, des ovulations ou des chaleurs qui marquent le rétablissement de 1'activité sexuelle. Notre protocole de contrôle ne nous a pas permis d'enregistrer les chaleurs post-partum. Celles-ci ont été observées par BARKER et WigGins (I964), MaUlÉon et DaUziER (I965), REsTali (I97I), LAND (I97I), sur différentes races et avec des fréquences variables, mais il s'agit en fait de chaleurs sans ovulation comme ces derniers l'ont observé.

En ce qui concerne la reprise de l'activité sexuelle, MaulÉon et Dauzier (I965) ont montré que chez les brebis Ile-de-France allaitant leurs agneaux en septembreoctobre, les ovulations se produisent bien avant que n'apparaissent les premières chaleurs (I à 3 cycles ovariens avant l'œstrus), de sorte que la première ovulation a lieu dans les 25 jours après la mise bas chez plus de 60 p. Ioo des brebis. Des observations comparables ont été faites par MILLER et WIGGINs dès Ig64 ou par HUNTER et Lishman (I967). Restal. (I97I) signale également des ovulations silencieuses Io à I 5 jours après la mise bas chez $70 \mathrm{p}$. Ioo des brebis Dorset Horn $\times$ Mérinos et remarque, en plus, que les corps jaunes qui accompagnent ces ovulations ont une " persistance " réduite de moitié (half normal life-span) et ne semblent pas sécréter de progestérone de façon sensible.

Une fois le comportement d'œestrus établi, on observe cependant des anomalies dans le cycle sexuel, en même temps qu'une fertilité réduite, comme cela est le cas avec nos Romanov dont la plupart, rappelons le, mettent bas pour la deuxième fois en moins de ro mois. C'est ainsi que dans une étude portant sur des brebis Finnoise, Dorset et croisées Finnois $\times$ Dorset, ayant mis bas au printemps et allaitant leurs agneaux, LAND (I97I) constate que les brebis des 3 races qui sont venues en chaleurs 3 à 56 jours après la mise bas, manifestent 2 catégories de cycles : des cycles courts de 7 jours (4 à 9) et des cycles normaux de $I 7$ jours ( 15 à 20), ces derniers étant cependant plus nombreux, contrairement à ce que nous observons avec nos Romanov. Avec 38 brebis Karakul agnelant de décembre à février (en Afrique du Sud) et après séparation des agneaux à la naissance, NEL (I965) montre que le premier cestrus apparaît en moyenne 27 jours après la mise bas ( 2 à 70 jours), mais que l'intervalle mise bas-fécondation est de 48 jours ( 23 à 87 jours), I3 p. Ioo seulement des brebis étant gravides au Ier ostrus. En I 968 LE Roux et NEL, obtiennent des résultats tout à fait comparables. Par ailleurs, ces 2 auteurs (NEL, I965; LE Roux et NEL, I968) signalent sans plus de précisions, une certaine tendance à l'apparition de cycles irréguliers après les premières chaleurs et ceci, jusqu'à 6 semaines après la mise bas. Enfin, au cours d'une expérimentation en rythme accéléré avec des brebis Dorset, Rambouillet et croisées, WhITfMan et al., (r972) constatent que parmi les brebis qui agnelent à l'automne (septembre à novembre), $85 \mathrm{p}$. Ioo reviennent en chaleurs et 7 I p. Ioo seulement sont fécondées, le premier œstrus et la fécondation ayant lieu respectivement 32 et 44 jours après la mise bas.

Tous ces résultats confirment donc que, si le comportement sexuel reprend 
assez rapidement après la mise bas, le pourcentage de fécondation est faible au cours du premier mois. Dans leur essai de synchronisation des chaleurs pendant l'ancestrus post-partum chez les brebis Ile-de-France et Préalpes, Thimonien et al., (Ig68) arrivent aussi à la conclusion que le pourcentage de mise bas à l'œstrus induit est faible peu après l'agnelage et augmente avec 1'intervalle parturition-début de traitement pour les brebis "traitées, comme il augmente avec l'intervalle parturitionsaillie pour les brebis " témoins ». Cette mauvaise fertilité peut être liée à l'état utérin qui empêche l'implantation trop tôt après la parturition (cf. notamment THImonier et al., I968 ; VANDEPLASSche, I968). Cependant, les observations de RESTALI, (I97I) sur le fonctionnement anormal du corps jaune, de LAND (I97I), LE Roux et NEI, (1968) et de nous-mêmes sur la présence de cycles courts à la reprise de l'activité sexuelle-cycles courts qui ont également été observés endéb ut et en fin de saison sexuelle par Wiliriams et al., I956 - prouvent qu'à cette période les mécanismes endocriniens qui influencent le fonctionnement de l'ovaire et l'état du tractus génital ne sont pas encore bien établis.

Rę̧u pour publication en avril 1973.

\section{REMERCIEMENTS}

Nous remercions MM. MaulÉon et Thimonier (Station de Recherches sur la Physiologie de la Reproduction, C. R. de Tours) et M. ThÉriez (Station de Recherches sur l'Élevage des Ruminants, C. R. V. Z. de Theix), des critiques et suggestions qu'ils nous ont apportées à la lecture de ce mémoire.

\section{SUMMARY}

\section{POST-PARTUM ANESTRUS IN ROMANOV EWES AFTER LAMBING} DURING THE BREEDING SEASON

On 46 Romanov ewes, subjected to an accelerated rhythm of reproduction (TCHAMITCHIAN et al., 1973), which had lambed between september I8th and october 23 rd I969 and which had been separated from their lambs some days afterwards, we recorded the onset of oestrus and practised matings during all heats observed till november zoth, i 969 . Two ewes never exhibited cestrus.

The first oestrus appeared I I to 47 days after lambing ( 24 days, on an average, for 44 ewes), but fertility was very low (fig. I). On the other hand, the distribution of the intervals between the first two ostrusses and between the second and the third one, shows the existence of short cycles of less than I I days and normal cycles of I 3 to 20 days (fig. I). Matings after a short cycle were less fertile than after a normal cycle (tabl. I). Thus conceptions took place 20 to 50 days after lambing ( 36 days on an average), but mainly between 34 and 45 days.

The interval between Ist cestrus and lambing, or between lambing and conception significantly varied according to the date of lambing, which seems to indicate that the post-partum ancestrus is minimum after october lambing (tabl. 2).

\section{RÉFÉRENCES BIBLIOGRAPHIQUES}

Barker H. B., Wiggins E. L., I964. Occurrence of post partum cestrus in fall lambing ewes. J. Anim. Sci., 23, 967-972.

Desvignes A., 1971. La race ovine Romanov. Revue bibliographique. Ann. Zootech., 20, 353-370. 
Hunter G. L., I968. Increasing the frequency of pregnancy in sheep. I. Some factors affecting rebreeding during the post partum period. Anim. Breed. Abst., 36, 347-378.

Hunter G. L., Ltshman A. W., I967. Post partum ovulation and ostrus in spring-lambing ewes. $J$. Reprod. Fert., 14, 473-475.

LAND R. B., I97I. The performance of Finn-Dorset sheep allowed to mate four times in two years. Anim. Prod., 13, 637-64r.

Mauléon P., Dauzier L., I965. Variations de durée de l'ancestrus de lactation chez les brebis de race Ile-de-France. Ann. Biol. anim. Bioch. Biophys., 5, 131-143.

Miller W. W., Wiggins E. L., r964. Ovarian activity and fertility in lactating ewes.. J. Anim. Sci., 23, $981-983$.

Nel J. A., I965. Post partum sex activity in Karakul sheep. Proc. S. Afr. Soc. anim. Prod., 4, 200204.

Restall B. J,, r97I. The effect of lamb removal on reproductive activity in Dorset $\times$ Merino ewes after lambing. J. Reprod. Fert., 24, I45-I46.

LE Roux P. J., NeL J. W., I968. The effect of supplementary feeding on post partum sex activity of Karakul ewes. Proc. S. Afr. Soc. anim. Prod., 7, I4I-I43.

Tchamtchian L., Ricordeau G., Lefèvere C., Desvignes A., I973. Performances des brebis Romanov soumises à un rythme accéléré de reproduction. Ann. Zootech. 22, 303-3ro.

Thimonier J., Mauléon P., Cognie Y., Ortavant R., ig68. Déclenchement de l'oestrus et obtention de la gestation pendant l'anostrus post partum chez les brebis à l'aide d'éponges vaginales imprégnées d'acétate de fluorogestone. Ann. Zootech., 17, 257-273.

VANDEPLASSche M., I968. La mortalité embryonnaire et son diagnostic. VIe Congr. intern. Reprod. Anim. Insém, artif., Paris, 1, 347-39I.

Whiteman J. V., Zollinger W. A., Thrift F. A., Gould M. B., i972. Post partum mating performance of ewes involved in a twice-yearly lambing program. $J$. Anim. Sci., 35, 836-842.

Williams S. M., Garrigus U.S., Norton H. W., Nalbandov A. V., 1956. Variations in the length of oestrus cycles and the breeding season in ewes. J. Anim. Sci., 15, 984-989. 